\title{
Unidirectionally coupled synchronization and circuit design of a novel chaotic system
}

\author{
Wei Xue ${ }^{1}$, Xue $\mathbf{L i}^{2}$ \\ ${ }^{1}$ Department of Automation, Tianjin University of Science and Technology, \\ 1038 Dagunanlu Road, Hexi Distric, Tianjin 300222, PR China \\ E-mail:xuewei@tust.edu.cn \\ ${ }^{2}$ Department of Automation, Tianjin University of Science and Technology, \\ 1038 Dagunanlu Road, Hexi Distric, Tianjin 300222, PR China \\ E-mail: lixueTJKJ@163.com
}

\begin{abstract}
In this paper, a novel three-dimensional autonomous chaotic system is proposed. Based on Lyapunov stability theory, a kind of unidirectionally coupled synchronization method is investigated. When coupling coefficient is selected properly, the response system can be synchronized completely with drive system. On this basis, the analog synchronization circuit of the system is designed and realized by using Multisim software. The obtained circuit simulation results are consistent with the numerical simulation results, which can further confirm the effectiveness and feasibility of the presented synchronization scheme.
\end{abstract}

Keywords: Novel chaotic system; Unidirectionally coupled synchronization; Numerical simulation; Circuit design.

\section{Introduction}

In 1963, American meteorologist Lorenz discovered chaos in a simple system of three autonomous ordinary differential equations, called the Lorenz system. ${ }^{1}$ As the first chaotic model, the Lorenz system has become a paradigm of chaos research. Recently, there has been increasing enthusiasm in finding new chaotic attractors, such as Chen system, ${ }^{2}$ Lü system, ${ }^{3}$ and so on. ${ }^{4-5}$ In this paper, a novel three-dimensional autonomous chaotic system is introduced. Numerical analysis shows that the proposed chaotic attractor is a new attractor, which is not topologically equivalent to the original chaotic system, or the Lorenz system, or the Chen system, or even the Lorenz system family.

Since the ideal of synchronizing chaotic systems was proposed by Pecora and Carroll, ${ }^{6}$ it has been an active research topic, mainly due to their practical applications in the fields of secure communication, image encryption, electrical engineering and so on. Many synchronization approaches have been proposed in order to synchronize the chaotic systems. For example, active synchronization, ${ }^{7}$ adaptive synchronization, ${ }^{8}$ projective synchronization, ${ }^{9}$ and sliding mode synchronization. ${ }^{10}$ A kind of unidirectionally coupled synchronization of the novel three-dimensional chaotic system is investigated. The suitable coupling coefficient is selected for the response system to realize the unidirectionally coupled synchronization. By means of theoretical analysis and numerical simulation verify the effectiveness of the presented synchronization scheme. On this basis, the analog synchronization circuit of the system is designed. 


\section{The novel chaotic system}

A novel three-dimensional autonomous chaotic system is proposed, which can be expressed as the following form:

$$
\left\{\begin{array}{l}
\dot{x}=a y-a x-y z \\
\dot{y}=b x+y+x z \\
\dot{z}=-d z+c y^{2}
\end{array}\right.
$$

where the parameters $a=33, b=50, c=3$, and $d=15$ are fixed, the phase portraits of system(1) are shown in Fig.1. The Lyapunov exponents of system (1) are calculated as $L_{1}=5.7673, L_{2}=0.0034$, and $L_{3}=-7.8236$.

The Lyapunov exponent spectrum of the $\operatorname{system}(1)$ varies with the increase of $d$ is given in Fig.2.
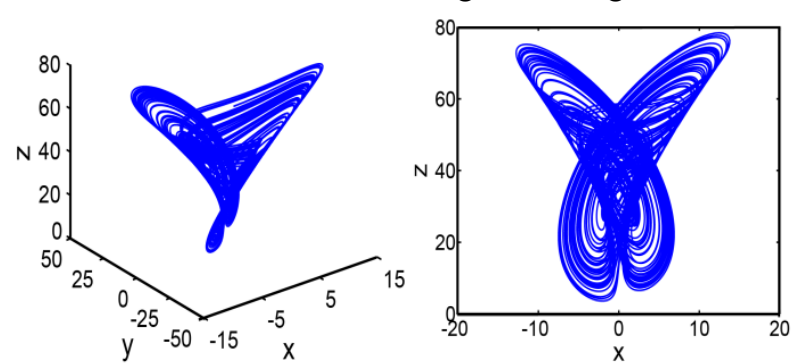

Fig.1. Phase portraits of system (1) with $a=33, b=50$, $c=3$ and $d=15$.

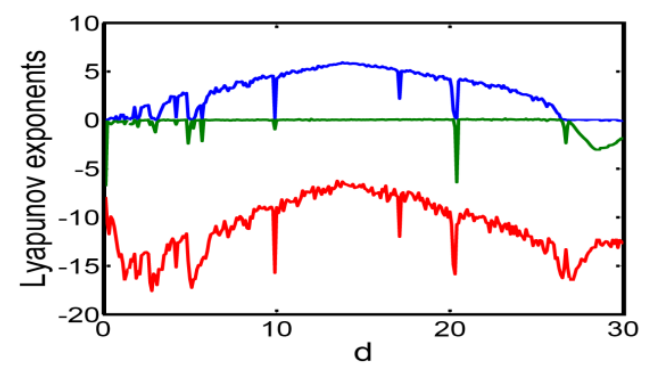

Fig.2. Lyapunov exponent spectrum of system (1) when $d \in(0,30)$

\section{Unidirectionally coupled synchronization of the chaotic system}

In order to realize the unidirectionally coupled synchro -nization of the system, the drive system is defined as follow:

$$
\left\{\begin{array}{l}
\dot{x}_{1}=a x_{2}-a x_{1}-x_{2} x_{3} \\
\dot{x}_{2}=b x_{1}+x_{2}+x_{1} x_{3} \\
\dot{x}_{3}=-d x_{3}+c x_{2} x_{2}
\end{array}\right.
$$

and the response system can be described in the form of:

$$
\left\{\begin{array}{l}
\dot{y}_{1}=a y_{2}-a y_{1}-y_{2} y_{3}-k\left(y_{1}-x_{1}\right) \\
\dot{y}_{2}=b y_{1}+y_{2}+y_{1} y_{3}-k\left(y_{2}-x_{2}\right) \\
\dot{y}_{3}=-d y_{3}+c y_{2} y_{2}-k\left(y_{3}-x_{3}\right)
\end{array}\right.
$$

Where $e_{1}=y_{1}-x_{1}, e_{2}=y_{2}-x_{2}$, and $e_{3}=y_{3}-x_{3}$.

And $k$ is the coupling coefficient, it can make system(2) and system(3) compose a coupling system.

From Eq.(2) and Eq.(3), the error system is obtained which can be expressed as:

$$
\left\{\begin{array}{l}
\dot{e}_{1}=a e_{2}-a e_{1}-y_{2} e_{3}-y_{3} e_{2}+e_{2} e_{3}-k e_{1} \\
\dot{e}_{2}=b e_{1}+e_{2}+y_{1} e_{3}+y_{3} e_{1}-e_{1} e_{3}-k e_{2} \\
\dot{e}_{3}=-d e_{3}+c y_{2} e_{2}+c y_{2} e_{2}-c e_{2} e_{2}-k e_{3}
\end{array}\right.
$$

Eq.(4) utilizes Laplace transform, the error system is provided which is described by:

$$
\left\{\begin{aligned}
s E_{1}(s)-e_{1}(0) & =a E_{2}(s)-a E_{1}(s)-L\left\{y_{2} e_{3}\right\} \\
& -L\left\{y_{3} e_{2}\right\}+L\left\{e_{2} e_{3}\right\}-k E_{1}(s) \\
s E_{2}(s)-e_{2}(0) & =b E_{1}(s)+E_{2}(s)+L\left\{y_{1} e_{3}\right\} \\
& +L\left\{y_{3} e_{1}\right\}-L\left\{e_{1} e_{3}\right\}-k E_{2}(s) \\
s E_{3}(s)-e_{3}(0) & =-d E_{3}(s)+c L\left\{y_{2} e_{2}\right\}+c L\left\{y_{2} e_{2}\right\} \\
& -c L\left\{e_{2} e_{2}\right\}-k E_{3}(s)
\end{aligned}\right.
$$

From Eq.(5), the error system is obtained as follow:

$$
\left\{\begin{aligned}
E_{1}(s)= & \frac{a E_{2}(s)}{s+k+a}-\frac{e_{1}(0)}{s+k+a}-\frac{L\left\{y_{2} e_{3}\right\}}{s+k+a} \\
& -\frac{L\left\{y_{3} e_{2}\right\}}{s+k+a}+\frac{L\left\{e_{2} e_{3}\right\}}{s+k+a} \\
E_{2}(s)= & \frac{b E_{1}(s)}{s+k-1}+\frac{e_{2}(0)}{s+k-1}+\frac{L\left\{y_{1} e_{3}\right\}}{s+k-1} \\
& +\frac{L\left\{y_{3} e_{1}\right\}}{s+k-1}-\frac{L\left\{e_{1} e_{3}\right\}}{s+k-1} \\
E_{3}(s)= & -\frac{e_{3}(0)}{s+k+d}+\frac{c L\left\{y_{2} e_{2}\right\}}{s+k+d}+\frac{c L\left\{y_{2} e_{2}\right\}}{s+k+d} \\
& -\frac{c L\left\{e_{2} e_{2}\right\}}{s+k+d}
\end{aligned}\right.
$$


Based on the final value theorem of Laplace transform, the error system is described in the form of:

$$
\begin{aligned}
\lim _{t \rightarrow \infty} e_{1}(t) & =\lim _{s \rightarrow 0} s E_{1}(s)=\frac{a}{s+k+a} \lim _{t \rightarrow \infty} e_{2}(t)-\lim _{s \rightarrow 0} \frac{s L\left\{y_{2} e_{3}\right\}}{s+k+a} \\
& +\lim _{s \rightarrow 0} \frac{s L\left\{y_{3} e_{2}\right\}}{s+k+a}+\lim _{s \rightarrow 0} \frac{s L\left\{e_{2} e_{3}\right\}}{s+k+a} \\
& =0 \\
\lim _{t \rightarrow \infty} e_{2}(t) & =\lim _{s \rightarrow 0} s E_{2}(s)=\frac{b}{s+k-1} \lim _{t \rightarrow \infty} e_{1}(t)+\lim _{s \rightarrow 0} \frac{s L\left\{y_{1} e_{3}\right\}}{s+k-1} \\
& +\lim _{s \rightarrow 0} \frac{s L\left\{y_{3} e_{1}\right\}}{s+k-1}-\lim _{s \rightarrow 0} \frac{s L\left\{e_{1} e_{3}\right\}}{s+k-1} \\
& =0 \\
\lim _{t \rightarrow \infty} e_{3}(t) & =\lim _{s \rightarrow 0} s E_{3}(s)=c \lim _{s \rightarrow 0} \frac{s L\left\{y_{2} e_{2}\right\}}{s+k+d}+c \lim _{s \rightarrow 0} \frac{s L\left\{y_{2} e_{2}\right\}}{s+k+d} \\
& -c \lim _{s \rightarrow 0} \frac{s L\left\{e_{2} e_{2}\right\}}{s+k+d} \\
& =0
\end{aligned}
$$
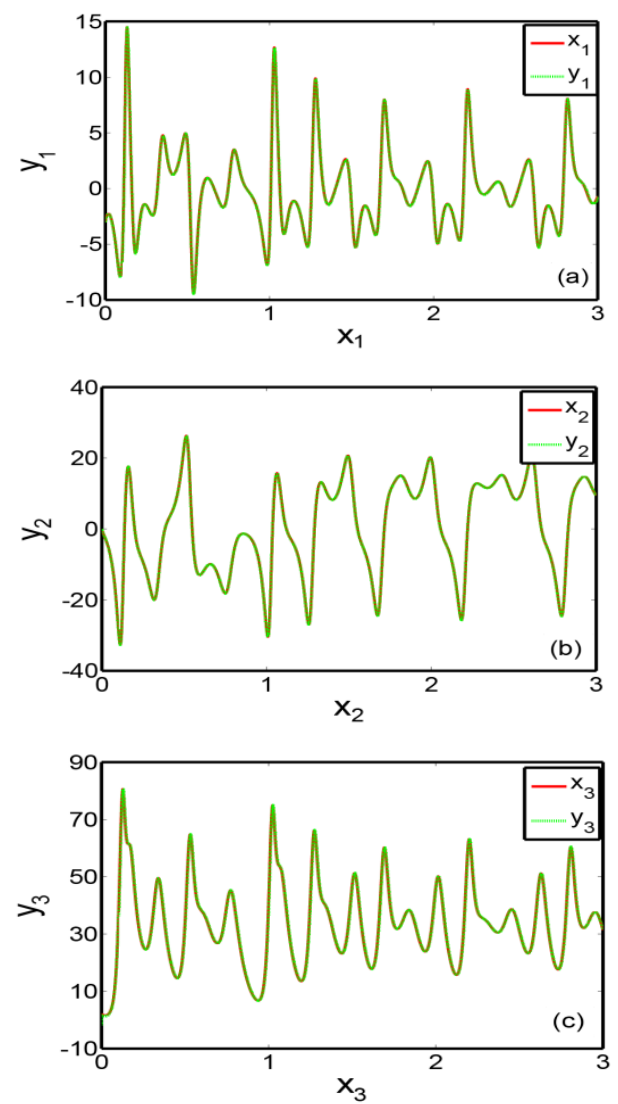

Fig.3. Variation of state variables with time: (a) $\left(x_{1}, y_{1}\right)$; (b) $\left(x_{2}, y_{2}\right)$; (c) $\left(x_{3}, y_{3}\right)$.
From Eq.(7), Eq.(8), and Eq.(9), the error system will be asymptotically stable, which means that $\lim e_{i}(t)=0(i=1,2,3)$. Theoretical analysis demonstrate system(2) can synchronize with system(3) completely.

\section{Numerical simulation}

In following, numerical simulation of unidirectionally coupled synchronization through designing the appropriate coupling coefficient is illustrated. The system parameters are chosen as $a=33, b=50, c=3$, and $d=15$. The initial values of the drive $\operatorname{system}(2)$ and the response $\operatorname{system}(3)$ are taken as $x_{1}(0)=-3, x_{2}(0)=0$, $x_{3}(0)=2, y_{1}(0)=-3, y_{2}(0)=-0.6$, and $y_{3}(0)=-1.6$, respectively. When the coupling coefficient is taken as $k=200$, it can be observed that the drive system and the response system are in asymptotic synchronization in Fig.3. In addition, curves of state variable are displayed in Fig.4. From Fig.4, we can observe that the curve is a straight line through the origin and $x_{i}=y_{i}(i=1,2,3)$. The obtained numerical simulation results agree with theoretical analysis results.
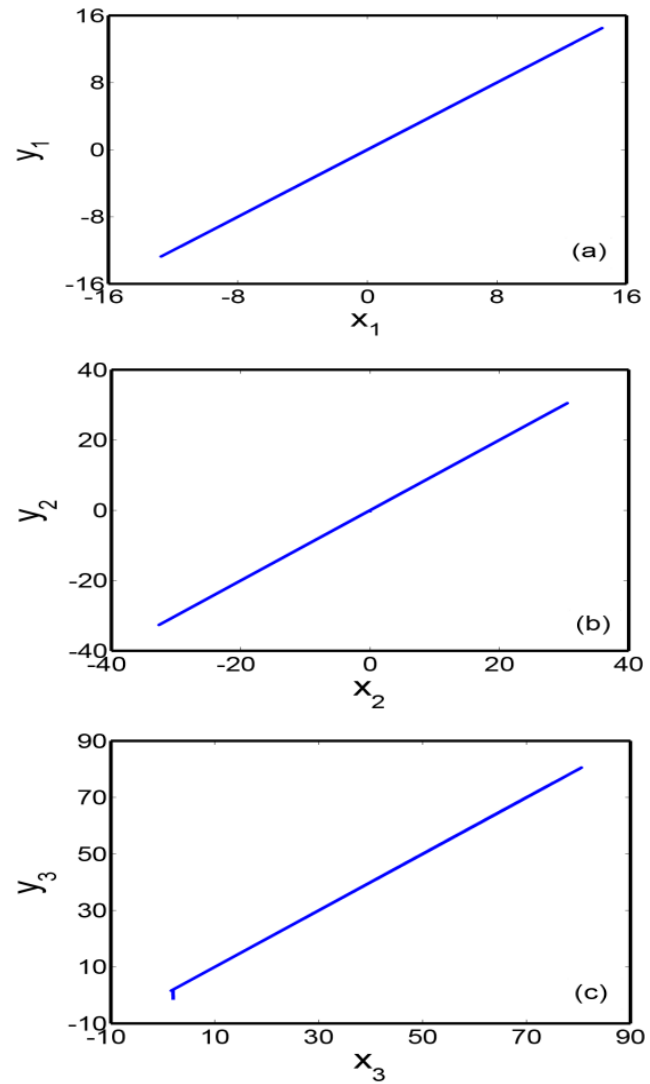

Fig.4. curves of state variable (a) $\left(x_{1}, y_{1}\right)$; (b) $\left(x_{2}, y_{2}\right)$; (c) $\left(x_{3}, y_{3}\right)$ 


\section{Synchronization circuit design}

In order to design the synchronous circuit, LF347N is selected as the amplifier, AD633JN is chosen as the multiplier and the power supply is $\pm 15 \mathrm{~V}$. Because of the restriction in the operating voltage of amplifier and multiplier, the state variable are decreased by 10 times, which means $\left(x_{1}, x_{2}, x_{3}\right) \rightarrow\left(10 x_{2}, 10 x_{2}, 10 x_{3}\right)$ and $\left(y_{1}, y_{2}, y_{3}\right) \rightarrow\left(10 y_{2}, 10 y_{2}, 10 y_{3}\right)$.

For the drive system, the circuit equation is as follows:

$$
\left\{\begin{array}{l}
\dot{x}_{1}=-\frac{1}{R_{1} C_{1}} x_{1}-\frac{1}{R_{2} C_{1}}\left(-x_{2}\right)-\frac{1}{10 R_{3} C_{1}} x_{2} x_{3} \\
\dot{x}_{2}=-\frac{1}{R_{4} C_{2}}\left(-x_{1}\right)-\frac{1}{R_{5} C_{2}}\left(-x_{2}\right)-\frac{1}{10 R_{6} C_{2}}\left(-x_{1}\right) x_{3} \\
\dot{x}_{3}=-\frac{1}{R_{7} C_{3}} x_{3}-\frac{1}{10 R_{8} C_{3}}\left(-x_{2}\right) x_{2}
\end{array}\right.
$$

The circuit equation of the response system is as follow:

$$
\left\{\begin{array}{l}
\dot{y}_{1}=-\frac{1}{R_{15} C_{4}} y_{1}-\frac{1}{R_{16} C_{4}}\left(-y_{2}\right)-\frac{1}{R_{17} C_{4}}\left(-x_{1}\right)-\frac{1}{10 R_{18} C_{4}} y_{2} y_{3} \\
\dot{y}_{2}=-\frac{1}{R_{19} C_{5}} y_{2}-\frac{1}{R_{20} C_{5}}\left(-y_{1}\right)-\frac{1}{R_{21} C_{5}}\left(-x_{2}\right)-\frac{1}{10 R_{22} C_{5}}\left(-y_{1}\right) y_{3} \\
\dot{y}_{3}=-\frac{1}{R_{23} C_{6}} y_{3}-\frac{1}{R_{24} C_{6}}\left(-x_{3}\right)-\frac{1}{10 R_{25} C_{6}}\left(-y_{2}\right) y_{2}
\end{array}\right.
$$

When $a=33, b=50, c=3$ and $d=15$, the circuit schematic of synchronization system is given in Fig.5. Where $R_{5}=1000 \mathrm{k} \Omega, \quad R_{7}=66.67 \quad \mathrm{k} \Omega, \quad R_{15}=4.29 \quad \mathrm{k} \Omega$, $R_{23}=4.65 \mathrm{k} \Omega, R_{4}=R_{20}=20 \mathrm{k} \Omega, R_{8}=R_{25}=3.33 \mathrm{k} \Omega$, $R_{1}, R_{2}, R_{16}=30.3 \mathrm{k} \Omega, \quad R_{17}, R_{19}, R_{21}, R_{24}=5 \quad \mathrm{k} \Omega$, $R_{3}, R_{6}, R_{9}, R_{10}, R_{11}, R_{12}, R_{13}, R_{14}, R_{18}, R_{22}, R_{26}, R_{27}, R_{28}, R_{29}$ $=10 \mathrm{k} \Omega, C_{1}, C_{2}, C_{3}, C_{4}, C_{5}, C_{6}=10 \mathrm{nF}$. The value of $R_{17}, R_{21}$ and $R_{24}$ of circuit schematic in respond system correspond to coupling coefficient $k$. The values of $R_{1}, R_{4}, R_{8}$ and $R_{7}$ of circuit schematic in drive system correspond to the value of $a, b, c$ and $d$, while we also can change the values of $a, b, c$ and $d$ by adjusting $R_{15}, R_{20}, R_{25}$ and $R_{23}$ of circuit schematic in respond system. The synchronization circuit result is shown in Fig. 6 through the oscilloscope TDS2014B is consistent with the numerical simulation result. From Fig.6, we can observe that the curve is a straight line through the origin and $x_{i}=y_{i}(i=1,2,3)$, indicating that $\operatorname{system}(2)$ and system(3) achieve synchronization completely.

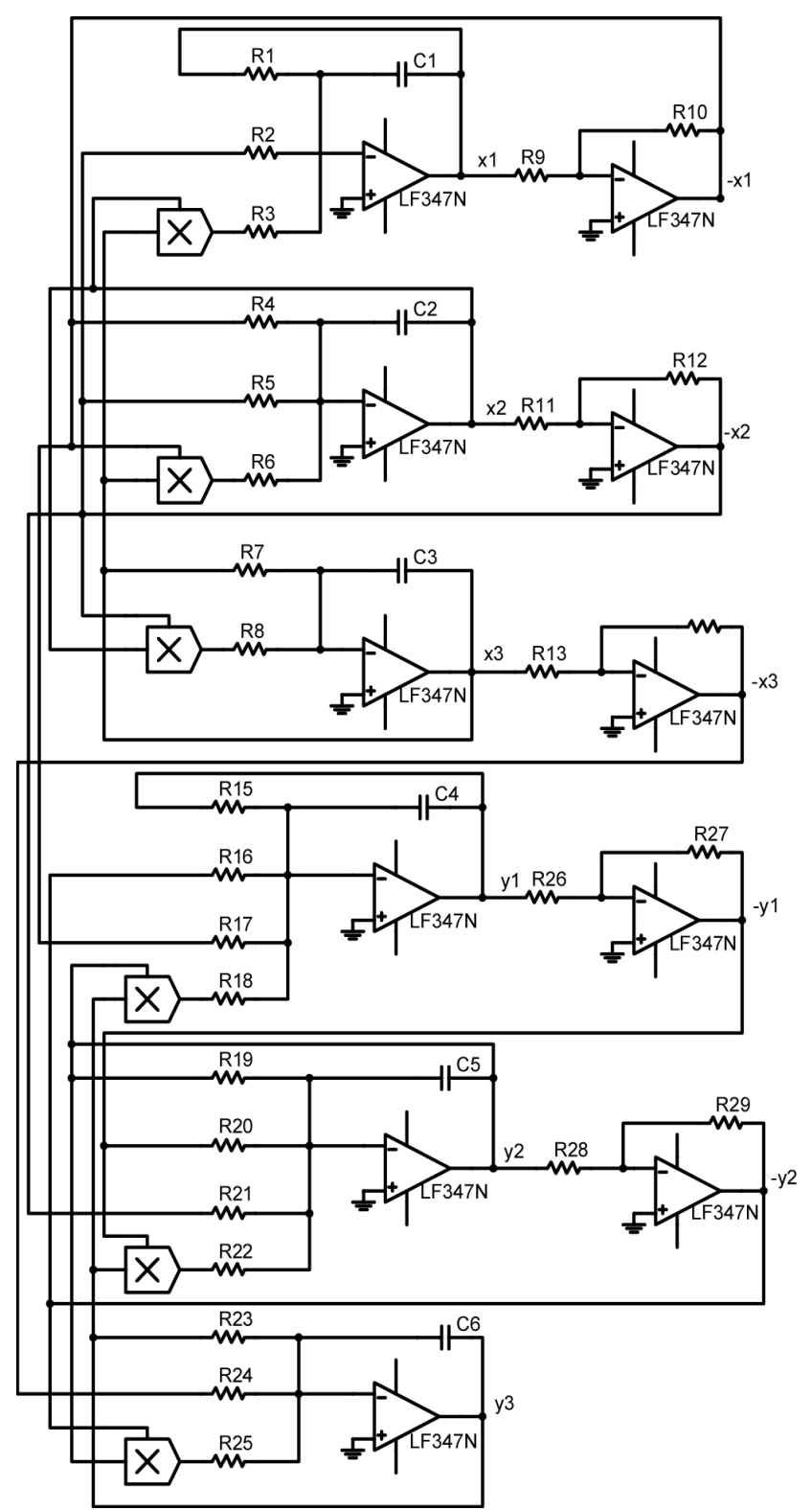

Fig.5. Circuit diagram of synchronization system 

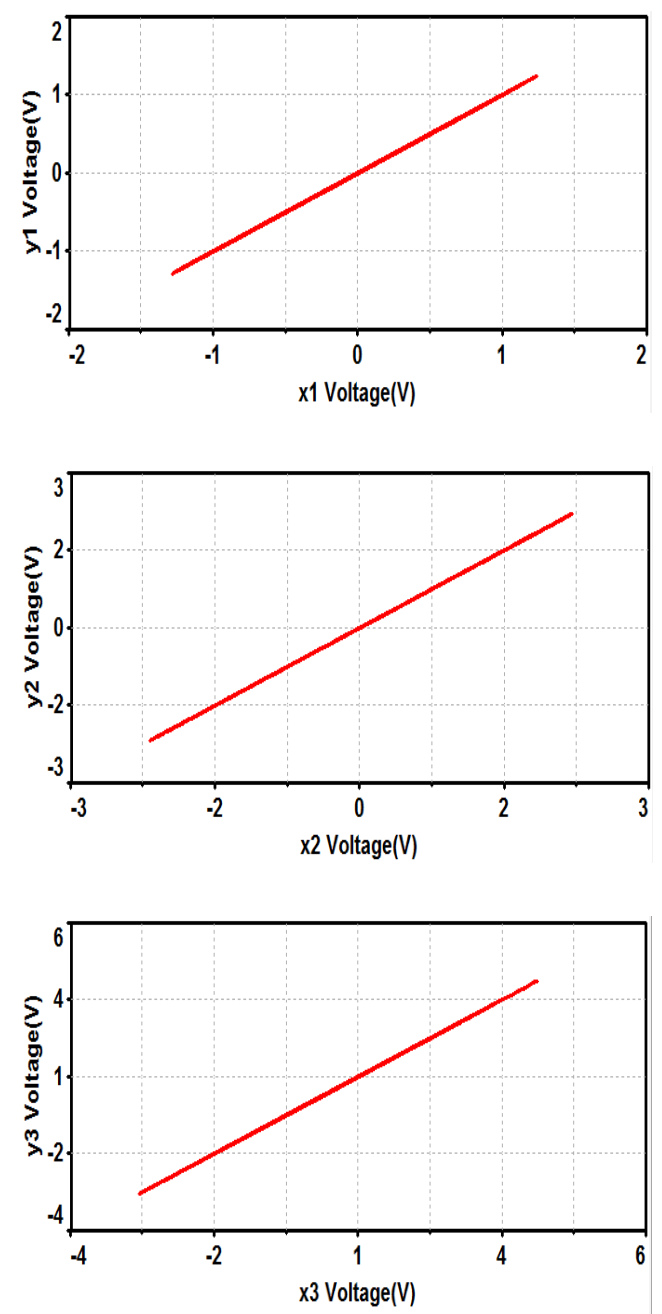

Fig.6. circuit simulation diagram (a) $\left(x_{1}, y_{1}\right)$; (b) $\left(x_{2}, y_{2}\right)$; (c) $\left(x_{3}, y_{3}\right)$.

\section{Conclusion}

In this paper, a novel three-dimensional autonomous chaotic system is discussed. The synchronization of the proposed chaotic system through selecting suitable coupling coefficient is investigated. The presented synchronization method is simple and the coupling coefficient is adjusted easily. On this basis, the analog synchronization circuit of the system is designed and realized by Multisim software. The circuit simulation results are coincide well with the theoretical analyses and the numerical simulation results, which can further verify the effectiveness and feasibility of the proposed synchronization scheme.

\section{Acknowledgement}

This work is supported by the Young Scientists Fund of the National Natural Science Foundation of China (Grant No.11202148).

\section{References}

1. E. N. Lorenz. Deterministic nonperiodic Flow, Journal of the Atmosphere Science. 20(2) (1963) 130-141.

2. G. R . Chen, T. Ueta. Yet another chaotic attractor, International Journal of Bifurcation and Chaos. 9(7) (1999) 1465-1466.

3. J. H. Lü, G. R. Chen. A new chaotic attractor coined, International Journal of Bifurcation and Chaos. 12(3) (2002) 659-661.

4. C. X. Liu, L. Liu, T. Liu, et al. A new butterfly-shaped attractor of Lorenz-like system, Chaos, Solitons and Fractals. 28(5) (2006) 1196-1203.

5. G. Y. Qi, S. Z. Du, G. R. Chen, et al. On a 4dimensional chaotic system, Chaos, Solitons and Fractals. 23(3) (2005) 1671-82.

6. L. M. Pecora, T. L. Carroll. Synchronization in chaotic system, Phys Rev Letter. 64(8) (1990) 821-824.

7. G. H. Li. An active control synchronization for tow modified chua circuits. Chinese Physics B. 14(3) (2005) 472-475.

8. J. H. Kim, C. W. Park, E. Kim, et al. Adaptive synchronization of T-S fuzzy chaotic systems with unknown parameters, Chaos, Solitons and Fractals. 24(4) (2005) 1335-1361.

9. J. Meng, X. Y. Wang. Generalized projective synchronization of a class of delayed neural networks. Physics Letters A. 22(3) (2008) 181-190.

10. H. T. Yau, J. J. Yan. Design of sliding mode controller for Lorenz chaotic system with nonlinear input, Chaos, Solitons and Fractals. 19(4) (2004) 891-898. 